\title{
MARKOV PROCESSES AND MARTIN BOUNDARIES
}

\author{
BY HIROSHI KUNITA AND TAKESI WATANABE
}

Communicated by J. L. Doob, December 31, 1962

1. Introduction. The theory of Martin boundaries associated with Markov processes has been established for the two typical and simplest classes; Brownian motions $[2 ; 6]$ and Markov chains on a denumerable space $[3 ; 5 ; 9]$. The purpose of this note is to give some general conditions under which one can construct the Martin boundary and derive the Martin representation of excessive functions, ${ }^{1}$ generalizing the method in [6] and [9]. Either of our conditions ((C), (D) or (E)) is satisfied, for example, by sufficiently wide classes of diffusion processes and space-time processes as well as by the two classes cited above and so our results can be connected with and applied to some subjects in analysis such as differential equations or convolution transforms.

Two different cases are discussed separately. One of them ( $\$ 3)$ is for the class of Markov processes such that there is a potential kernel of function type. In this case we shall also show a method of determining the potential kernel of function type which is somewhat different from Hunt's method in [4, III]. The other case ( $\$ 4)$ is for the class of regular step processes [10] (including Markov chains) with the step measures having density functions. A full proof will appear elsewhere.

2. Definitions and notations. Let $S$ be a locally compact, noncompact, separable Hausdorff space and $X$, a temporally homogeneous Markov process on $S$ satisfying Hunt's condition (A) [4, I, pp. 4850]. Following [1], such $X$ is called a Hunt process. For the details of the definition see $[1 ; 4 ; 7]$. Let $x_{t}$ denote the path functions of $X, P_{x}$ and $E_{x}$ the probabilities and expectations for $X$ starting at $x$ and $\sigma_{A}$ the hitting time for a subset $A$ of $S$, inf $\left\{t>0, x_{t} \in A\right\}$. We shall always assume that $X$ is transient: $P_{x}\left(\sigma_{K^{c}}<\infty\right)=1$ for every $x$ and every compact $K$ of $S$, where $K^{c}$ means the complement of the set $K$. For a measurable function $u$ defined on $S, E_{x}\left(u\left(x_{t}\right)\right)$ and $E_{x}\left(u\left(x_{\sigma_{A}}\right)\right)$ are denoted by $H_{t} u(x)$ and $H_{A} u(x)$ respectively. We shall say $u$ : s superharmonic if it is positive ${ }^{2}$ and if it satisfies $u(x) \geqq H_{A^{c}} u(x)$

${ }^{1}$ Meyer [7, Part II] also discussed this problem without introducing the boundary. His approach is based on Choquet's representation theorem for compact convex sets.

2 The word 'positive' is used in the sense of 'non-negative.' 
for every $x$ and every Borel subset $A$ with compact closure. In particular if equality holds in the above relation, $u$ is called harmonic. Any superharmonic function satisfies $u \geqq H_{t} u$ for every $t$ and any harmonic function is excessive (i.e., $u \geqq H_{t} u$ and $H_{t} u \rightarrow u$ as $t \rightarrow 0$ ). Let $\left\{K_{n}\right\}$ be a sequence of compact sets increasing to $S$. A function $u$ is said to be a potential if it is excessive and if $H_{K_{n}^{c}} u(x) \rightarrow 0$ as $n \rightarrow \infty$ for every $x$ such that $u(x)<+\infty$. An excessive function $u$ is said to be extreme if, whenever $u=u_{1}+u_{2}$ with $u_{1}$ and $u_{2}$ both excessive, each $u_{i}$ is a constant multiple of $u$.

Consider the $n$th jump time $\sigma_{n}(w)=\sigma_{n-1}(w)+\sigma_{1}\left(w_{\sigma_{n-1}^{+}}\right)^{3}$ with $\sigma_{1}(w)=\inf \left\{t>0, x_{t}(w) \neq x_{0}(w)\right\}$ and define $q(x)=\left[E_{x}\left(\sigma_{1}\right)\right]^{-1}$ and $\pi(x, A)=P_{x}\left(x_{\sigma_{1}} \in A\right)$. The point $x$ is called a sojourn state of $0<q(x)$ $<\infty$. A Hunt process $X$ is said to be a regular step process if all points of $S$ are sojourn states and if $\lim _{n \rightarrow \infty} \sigma_{n}$ is not smaller than the life time of $X$. We shall say a positive function $u$ is $\pi$-superharmonic if $u \geqq \pi u=\int \pi(\cdot, d y) u(y)$ and $\pi$-harmonic if $u=\pi u$. For a regular step process, the three notions "excessive," "superharmonic" and " $\pi$ superharmonic" coincide with each other. Any harmonic function is $\pi$-harmonic but some additional condition will be needed for the converse statement.

Finally we introduce notations for some function families on $S$; $\beta=$ the family of all bounded measurable functions on $S, B_{0}=\{f \in \Theta$ and $f$ vanishes outside of some compact set $\}, \mathbb{e}=$ the family of all bounded continuous functions on $S, \mathbb{C}_{0}=B_{0} \cap \mathcal{e}$ and $D_{0}=$ a countable family of functions in $\mathfrak{C}_{0}$ such that any function $f$ in $\mathfrak{C}_{0}$ is uniformly approximated by some sequence of functions in $D_{0}$ vanishing outside of the carrier of $f$.

3. When $X$ has a potential kernel of function type. Let $\xi$ be a $\sigma$-finite measure on $S$ with $\xi(G)>0$ for any open set $G, G_{\alpha}(x, A)$ $=\int_{0}^{\infty} e^{-\alpha t} P_{x}\left(x_{t} \in A\right) d t$ for $\alpha>0$ and $G_{\alpha} f(x)=\int f(y) G_{\alpha}(x, d y)$. We shall now introduce the condition (A) that $G_{\alpha} f \in \mathcal{C}$ for every $f$ of $B$ and that there are measures $G_{\alpha}^{*}(x, \cdot)$ defined on $S$ such that $G_{\alpha}^{*} f$

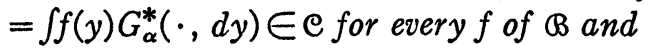

$$
\int f(x) G_{\alpha} g(x) \xi(d x)=\int g(x) G_{a}^{*} f(x) \xi(d x)
$$

for every $f, g$ of $B_{0}$ and, for every $f$ of $\mathfrak{C}, \alpha G_{\alpha}^{*} f$ converges boundedly and uniformly on any compact set to $f$ as $\alpha \rightarrow \infty$. Note that the measure $G_{\alpha}^{*}(x, \cdot)$ is unique if it exists. A positive and measurable

\footnotetext{
- The symbol $w^{+}{ }_{\sigma_{n-1}}$ denotes the shifted path defined by $x_{t}\left(w^{+}{ }_{\sigma_{n-1}}\right)=x_{t+\sigma_{n-1}}(w)$.
} 
function $f$ is said to be co-excessive if $\alpha G_{\alpha}^{*} f \leqq f$ and the left side increases to the right side as $\alpha \rightarrow \infty$. Since both $G_{\alpha}$ and $G_{\alpha}^{*}$ satisfy the resolvent equation, $G_{0}(x, A)=\lim _{\alpha \rightarrow 0} G_{\alpha}(x, A)$ and $G_{0}^{*}(x, A)=\lim _{\alpha \rightarrow 0} G_{\alpha}^{*}(x, A)$ give measures on $S$ for each $x$. Corresponding to Hunt's condition (G) $[4, \mathrm{III}]$, we introduce the condition (B) that both $G_{0}(x, A)$ and $G_{0}^{*}(x, A)$ is bounded in $x$ for any compact set $A$.

LemMA 1. Under the conditions (A) and (B) there exists uniquely the function $G(x, y)$ such that (i) $G_{0}(x, d y)=G(x, y) \xi(d y)$, (ii) $G_{0}^{*}(y, d x)$ $=G(x, y) \xi(d x)$, (iii) $G(\cdot, y)$ is excessive for each $y$ and (iv) $G(x, \cdot)$ is co-excessive for each $x$.

With the kernel $G(x, y)$ one can establish results like those obtained by Hunt $[4$, III, \$18]. We shall now state only the Riesz decomposition of excessive functions. For each $y, G(\cdot, y)$ is extreme and so it is either a potential or harmonic. Define $S_{P}=\{y ; G(\cdot, y)$ is a potential $\}$. $^{*}$ Every excessive function $u$, finite almost everywhere relative to the measure $\xi$, can be decomposed uniquely (including the determination of the measure $\nu$ ) in the form

$$
u=\int_{S_{P}} G(\cdot, y) \nu(d y)+(\text { a harmonic function }) .
$$

Let $\gamma$ be a $\sigma$-finite measure on $S$. To introduce the Martin boundary (relative to $\gamma$ ) we shall further assume either of the following conditions: (C) $\gamma G(y)=\int \gamma(d x) G(x, y)$ is strictly positive and continuous on $S$, allowing the value infinity or (D) the conditions (A) and (B) are satisfied for $\xi(A)=\int \gamma(d x) G_{0}(x, A)$. With (C) let $\kappa(x, y)$ be $G(x, y) / \gamma G(y)$ if $\gamma G(y)<\infty$ and zero if $\gamma G(y)=\infty$. With (D) the corresponding $G(x, y)$ is denoted by $\kappa(x, y)$. In both cases, $f_{\kappa}(y)$ $=\int f(x) \kappa(x, y) \xi(d x) \in \mathcal{C}$ for every $f$ of $\beta_{0}$. Let $\rho_{1}$ be the metric of one point compactification of $S$ and

$$
\rho_{2}\left(y, y^{\prime}\right)=\sum_{n=1}^{\infty} \frac{1}{2^{n}} \frac{\left|f_{n} \kappa(y)-f_{n} \kappa\left(y^{\prime}\right)\right|}{1+\left|f_{n} \kappa(y)-f_{n} \kappa\left(y^{\prime}\right)\right|},
$$

where $\left\{f_{n}\right\}=D_{0}$. The completion $M$ of $S$ by $\rho=\rho_{1}+\rho_{2}$ is called the Martin space and $\partial S=M-S$, the Martin boundary. Both $M$ and $\partial S$ are compact metric spaces and the relative topology of $S$ coincides with the original one. If $f \in \mathbb{C}_{0}, f \kappa(y)$ has a continuous extension to $M$, so that $f_{\kappa}(\eta), \eta \in \partial S$, determines uniquely a measure $\kappa(d x, \eta)$.

Lemma 2. $\kappa(x, y)$ is extended uniquely to $S \times M$ in such a way that, for each $\eta \in \partial S, \kappa(\cdot, \eta)$ is excessive and $\kappa(d x, \eta)=\kappa(x, \eta) \xi(d x)$.

4 If $\xi$ is an excessive measure $[4, I]$ of $X$, we have $S=S_{P}$. 
The reduced function of an excessive function may be defined for any Borel set of $M$. But, for short, we shall here confine ourselves to the simplest case. Let $D$ be a closed set of $\partial S,\left\{G_{n}\right\}$ a sequence of open sets in $M$ decreasing to $D$ and $\left[G_{n}\right]=G_{n} \cap S$. The function $\bar{H}_{D} u=\lim _{\alpha \rightarrow \infty} \alpha G_{\alpha}\left(\lim _{n \rightarrow \infty} H_{\left[G_{n}\right]} u\right)$ is called the reduced function of $u$ to $D$. Such a function is harmonic. The set of boundary points $\eta$ such that $\bar{H}_{\{\eta\}} u$ is not identically zero for some $\gamma$-integrable excessive function $u$ is denoted by $(\partial S)_{1}$ and the set $S_{P} \cap\left\{y_{i} \int \gamma(d x) \kappa(x, y)=1\right\}$, by $S_{1}{ }^{5}$ The set $M_{1}=S_{1} \cup(\partial S)_{1}$ is said to be the essential part of $M$. A point $\eta$ of $\partial S$ is in $(\partial S)_{1}$ if and only if $\int \gamma(d x) \bar{H}_{\{\eta\}} \kappa(x, \eta)=1$. If $\eta \in(\partial S)_{1}$, $\kappa(\cdot, \eta)$ is extreme and harmonic. Both $S_{1}$ and $(\partial S)_{1}$ are Borel sets of $M$.

Theorem 1. Suppose that the condition (C) or (D) is satisfied. Then every $\gamma$-integrable and excessive function $u^{6}$ can be represented uniquely in the form

$$
u(x)=\int_{M_{1}} \kappa(x, \eta) \mu(d \eta),
$$

using a bounded measure $\mu$ on $M_{1}$. The total mass of $\mu$ is concentrated on $(\partial S)_{1}$ if and only if $u$ is harmonic.

Here is an alternative classification of the essential part $M_{1}$, which implies a new approach to the boundary theory. We consider the case of the condition (D) being satisfied. Moreover we assume that $M$ is nothing but the completion of $S$ by $\rho_{2}$ above. ${ }^{7}$ Then there exists a Markov process $X^{\prime}$ defined on $M$ such that $\kappa(x, \cdot)$ is excessive relative to $X^{\prime}$ for each $x$ of $S$. Such $X^{\prime}$ need no longer be a Hunt process but a process such as was discussed by Ray [8]. The set $M-M_{1}$ coincides with the set of all branching points $[8, \mathrm{p} .45]$ of $X^{\prime}$.

4. The case of regular step processes. For a regular step process $X$, the assumptions of the preceding section are not satisfied unless $S$ is a denumerable space, so that we shall need a different treatment. In this section we are interested in the Martin representation of $\pi$-harmonic functions and harmonic functions for some class of regular step processes. First we assume that $\pi(x, A)$ is absolutely continuous relative to some $\sigma$-finite measure $m$ with $m(G)>0$ for any open set $G$. Let $\pi(x, y)$ be the density function, $\pi^{n}(x, y)=\int \pi^{n-1}(x, z) \pi(z, y) m(d z)$ and $G(x, y)=\sum_{n=1}^{\infty} \pi^{n}(x, y)$. The finiteness of $G(x, y)$ is also assumed.

${ }^{5}$ In case the condition (D) is satisfied, we have $S=S_{1}$.

6 Such $u$ is necessarily finite almost everywhere relative to $\xi$.

${ }^{7}$ It is expected that this assumption will be dropped. 
Let $\gamma$ be a measure such that $\gamma G(y)=\int \gamma(d x) G(x, y)$ is strictly positive on $S$. The function $\kappa(x, y)$ is defined as $G(x, y) / \gamma G(y)$ if $\gamma G(y)<\infty$ and as zero if $\gamma G(y)=\infty$. Finally we assume that $f_{\kappa}(y)$ $=\int f(x) \kappa(x, y) m(d x)$ belongs to $\mathfrak{C}$ whenever $f$ is in $\mathfrak{C}_{0}$. The above statements in italics are referred to as the condition (E). The Martin boundary $\partial S$, the reduced function $\vec{H}_{D} u$ and the essential part $(\partial S)_{1}$ of the boundary are defined in the same way as in the previous section. The definition of $k(x, \eta)$ for $\eta \in \partial S$ is as follows. As in the previous section to each point $\eta \in \partial S$ corresponds a measure $\kappa(d x, \eta)$ such that $\int f(x) \kappa(d x, \eta)$ coincides with the continuous extension of $f_{\kappa}(y)$ to the boundary for every $f$ in $\mathfrak{C}_{0}$. Define $\kappa(x, \eta)=\int \pi(x, y) \kappa(d y, \eta)$ for $\eta \in \partial S$. Such $\kappa(x, \eta)$ may depend on the choice of the version of the densities $\pi(x, y)$ satisfying the condition (E), while $\partial S$ and $(\partial S)_{1}$ do not so depend. Moreover, we are not sure that $\kappa(\cdot, \eta)$ is excessive. However, one can prove that, if $\eta \in(\partial S)_{1}, \kappa(d x, \eta)$ is absolutely continuous relative to $m(d x)$ and $\kappa(\cdot, \eta)$ is not only $\pi$-harmonic but also harmonic. Moreover, $(\partial S)_{1}$ is a Borel set of $\partial S$.

THEOREM 2. Suppose that the condition (E) is satisfied and $u$ is a $\gamma$-integrable function. Then the following three conditions are equivalent to each other. (i) $u$ is harmonic. (ii) $u$ is $\pi$-harmonic. (iii) $u$ is expressible in the form

$$
u(x)=\int_{(\partial S)_{1}} \kappa(x, \eta) \mu(d \eta),
$$

using a bounded measure $\mu$. Moreover the above integral representation is unique.

REMARK. If $X$ is of discrete time parameter (i.e., Markov chain), the condition (E) may be imposed to the transition function $H(x, A)$ instead of $\pi(x, A)$. But (E) is more natural to be imposed to $\pi$ than to $H$. Also note that $H(x, A)-\delta(x, A)=q(x)[\pi(x, A)-\delta(x, A)]$ and $H(x, A)=\pi(x, A)$ if $H(x,\{x\})=0$, where $\delta(x, A)$ denotes the unit measure at $x$.

\section{REFERENCES}

1. R. M. Blumenthal, R. K. Getoor and H. P. McKean, Jr., Markov processes with identical hitting distributions, Illinois J. Math. 6 (1962), 402-420.

2. M. Brelot, Le problème de Dirichlet. Axiomatique et frontière de Martin, J. Math. Pures Appl. 35 (1956), 297-335.

3. J. L. Doob, Discrete potential theory and boundaries, J. Math. Mech. 8 (1959), 433-458.

4. G. A. Hunt, Markoff processes and potentials. I, III, Illinois J. Math. I (1957), 44-93, 2 (1958), 151-213. 

340 .

5. - Markoff chains and Martin boundaries, Illinois J. Math. 4 (1960), 313-

6. R. S. Martin, Minimal positive harmonic functions, Trans. Amer. Math. Soc. 49 (1941), 137-172.

7. P. A. Meyer, Fonctionnelles multiplicatives et additive de Markov, Ann. Inst. Fourier (Grenoble) 12 (1962), 125-230.

8. D. Ray, Resolvents, transition functions and strongly Markovian processes, Ann. of Math. (2) 70 (1959), 43-78.

9. T. Watanabe, On the theory of Martin boundaries induced by countable Markov processes, Mem. Coll. Sci. Univ. Kyoto Ser. A 33 (1960), 39-108.

10. - On the equivalence of excessive functions and superharmonic functions in the theory of Markov processes. I, Proc. Japan Acad. 38 (1962), 397-401.

KYUSHU UNIVERSITY AND

UNIVERSITY OF ILLINOIS

\section{A NONTRIVIAL NORMAL SUP NORM ALGEBRA}

\section{BY ROBERT MCKISSICK ${ }^{1}$}

Communicated by I. M. Singer, December 17, 1962

Let $X$ be a compact Hausdorff space and $A$ a complex linear algebra of continuous complex-valued functions defined on $X$. Suppose $A$ is normal on $X$, i.e., for every pair of disjoint closed sets $K_{0}, K_{1}$ in $X$, there exists a function $f \in A$ such that $f\left(K_{0}\right)=0$ and $f\left(K_{1}\right)=1$. Does it follow that every continuous complex-valued function on $X$ can be uniformly approximated by functions in $A$ ? With the additional assumption that $A$ is closed under complex conjugation, it follows by the Stone-Weierstrass theorem. (Trivially, if $A$ is normal then $A$ separates points.) The same theorem implies that the analogous question in the case of real-valued functions has an affirmative answer. However, in the complex-valued case it need not be so. An example will be given which demonstrates this. In this example, the space $X$ is a suitably chosen compact set in the complex plane. The algebra is $R(X)$, the algebra of all functions which can be uniformly approximated on $X$ by rational functions whose poles lie outside $X$. It will be shown that $R(X)$ is normal on $X$ and is a proper sub-algebra of $C(X)$, the algebra of all continuous complex-valued functions on $X$. Since $R(X)$ is closed under uniform limits, this will be sufficient.

Two lemmas are needed to accomplish this. One is a modification of an observation of Mergelyan [1]. The second represents a slight extension of a result due to Beurling [2].

1 The research in this paper forms part of the author's doctoral dissertation, submitted to the Massachusetts Institute of Technology, Spring, 1963. 